\title{
Health-Related Quality of Life Assessments with Once-Weekly Glucagon-Like Peptide-1 Receptor Agonists in Type 2 Diabetes Mellitus
}

Liana K. Billings, MD, MMSc; Yehuda Handelsman, MD, FACP, FNLA, FASPC, MACE; Michael Heile, MD, FAAFP; Doron Schneider, MD, FACP; and Kathleen Wyne, MD, PhD, FACE, FNLA

\begin{abstract}
Type 2 diabetes (T2DM) is associated with significant impairment in healthrelated quality of life (HRQOL). A patient-centered collaborative approach is recommended to optimize clinical outcomes, including HRQoL, in this patient population. Glucagon-like peptide-1 receptor agonists (GLP-1 RAs) provide effective glycemic control and have demonstrated beneficial effects on HRQ 0 L and treatment satisfaction. Available once-weekly GLP-1 RAs may offer enhanced convenience compared with daily GLP-1 RAs and include exenatide extended-release (ER), dulaglutide, and semaglutide.

This article reviews the impact of once-weekly GLP-1 RAs on HRQoL and treatment satisfaction in patients with T2DM. Compared with oral antihyperglycemic drugs, insulin, and daily GLP-1 RAs, once-weekly GLP-1 RAs offer benefits with regard to HRQOL and treatment satisfaction. These benefits appear to be largely mediated by relative drug effects on glycemic control, weight, and hypoglycemia. While there was not an overall class benefit of once-weekly GLP-1 RAs compared with daily GLP-1 RAs on $\mathrm{HRQOL}$ and treatment satisfaction, results suggested that once-weekly GLP-1 RAs may enhance certain elements of treatment satisfaction and increase willingness to continue treatment. In 2 studies comparing onceweekly GLP-1 RAs with each other, semaglutide produced significantly greater improvement in overall treatment satisfaction compared with exenatide ER but not dulaglutide. Once-weekly GLP-1 RAs represent an effective and convenient treatment option that may potentially increase treatment satisfaction and enhance adherence, contributing to improved health outcomes.
\end{abstract}

J Manag Care Spec Pharm. 2018;24(9-a):S30-S41

Copyright @ 2018, Academy of Managed Care Pharmacy. All rights reserved.

$\mathrm{T}$ ype 2 diabetes (T2DM) is associated with significant impairments on a broad range of health-related quality of life (HRQoL) measures, including high rates of depression..$^{1-5}$ The concept of HRQoL takes into account the patient's perspectives of both physical and mental health. ${ }^{6}$ Diabetes management has undergone an increasing emphasis on the need to not just address clinical outcomes such as hyperglycemia but also to view improvement in HRQoL as a goal of treatment. ${ }^{6,7}$ In addition to the obvious benefits of improving patients' well-being and functioning, a positive impact of treatment on HRQoL may potentially lead to improvement in mood and to better lifestyle choices that translate to improvements in other health endpoints (e.g., glycemic control, obesity, blood pressure control, self-management, and education). Further, resulting improvements in physical function may lead to increased physical activity, which may improve health outcomes.

A patient-centered collaborative approach is recommended to optimize clinical outcomes and improve HRQoL in patients with T2DM. ${ }^{7}$ Both pharmacologic and nonpharmacologic approaches are important in this regard. Weight loss, associated with lifestyle changes or pharmacotherapy, is one key component of diabetes management that may also contribute to improvements in HRQoL and treatment satisfaction, although HRQoL has many determinants. ${ }^{8,9}$ There is a wide range of pharmaceutical treatment options available for the management of T2DM, including both injectable and oral medications. However, many treatment regimens are associated with weight and blood glucose fluctuations as well as with high costs and administration issues (e.g., frequent dosing and high pill-burden load) that may potentially impair HRQoL.

Once-daily and twice-daily (BID) glucagon-like peptide-1 receptor agonists (GLP-1 RAs) effectively reduce hyperglycemia and have demonstrated beneficial effects on HRQOL and treatment satisfaction..$^{7,10-14}$ This class of medication is also associated with reductions in body weight, which, along with other mechanisms, may contribute to improved psychological and emotional well-being, social/daily functioning, and health perceptions. ${ }^{10-12}$ In recent years, once-weekly GLP-1 RAs have become available, including exenatide extended-release (ER), dulaglutide, albiglutide, and semaglutide. This article reviews the impact of once-weekly GLP-1 RAs, oral antihyperglycemic drugs (OADs), insulin, or daily GLP-1 RAs on HRQoL and treatment satisfaction in patients with T2DM.

\section{Quality of Life and Treatment Satisfaction}

In order to enable meaningful interpretation of data on the effects of treatments on HRQoL and treatment satisfaction, it is critical to use validated tools to assess HRQoL in diabetes outcomes studies. ${ }^{6}$ Table 1 provides a summary of the tools used to evaluate HRQoL and treatment satisfaction in studies of onceweekly (QW) GLP-1 RAs. Of note, some studies have employed validated tools to specifically examine the impact of treatment with once-weekly GLP-1 RAs on weight-related HRQoL 27,28 and

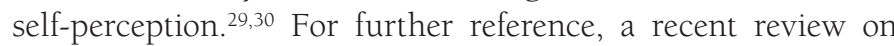
T2DM and HRQoL describes a broad range of HRQOL assessment tools used in patients with diabetes. ${ }^{6}$ Tables 2,3 , and 4 summarize the effects on HRQoL and treatment satisfaction with exenatide ER, dulaglutide, and semaglutide, respectively.

\section{Once-Weekly GLP-1 RAs Versus Placebo}

Compared with placebo, dulaglutide $0.75 \mathrm{mg}$ and $1.5 \mathrm{mg}$ as add-on therapy to metformin and pioglitazone in AWARD-1 significantly improved measures of patients' self-perception related to body 


\begin{tabular}{|c|c|c|}
\hline \multicolumn{2}{|c|}{ Assessment Tool } & Description of Tool \\
\hline \multicolumn{3}{|c|}{ Quality of life tools } \\
\hline APPADL & $\begin{array}{l}\text { Ability to Perform } \\
\text { Physical Activities of } \\
\text { Daily Living }\end{array}$ & $\begin{array}{l}\text { 7-item assessment of how difficult engagement in activities deemed integral to normal life is (e.g., walking, } \\
\text { standing, and climbing stairs); rated on 5-point numeric scale from "not at all difficult" to "unable to do"15 }\end{array}$ \\
\hline DHP-18 & $\begin{array}{l}\text { 18-item Diabetes Health } \\
\text { Profile }\end{array}$ & $\begin{array}{l}\text { 18-item questionnaire assessing psychological distress, barriers to activity, and disinhibited eating and } \\
\text { including disease-specific health-related quality of life questions } 16 \\
\text { Items are scored from } 0 \text { (never/not at all/very likely) to } 3 \text { (always/very often/very much/not at all likely) on a } \\
\text { 4-point Likert-type; raw scale scores on each subscale are transformed to an overall ("common") score ranging } \\
\text { from 0-100, with } 0 \text { indicating no dysfunction16 }\end{array}$ \\
\hline EQ-5D & EuroQol 5 Dimensions & $\begin{array}{l}\text { 2-part questionnaire } \\
5 \text { dimensions: mobility, self-care, usual activities, pain/discomfort, anxiety/depression } \\
\text { 3-level responses: no problem, some problem, extreme problem } \\
\text { Visual analog scale component rating daily health from 0-100 (worst to best health imaginable) }{ }^{17}\end{array}$ \\
\hline IWQOL-Lite & $\begin{array}{l}\text { Impact of Weight on } \\
\text { Quality of Life-Lite }\end{array}$ & $\begin{array}{l}\text { Questionnaire assessing effect of weight on various areas of life; brief version relating identified scales to } \\
\text { impact on physical function (11 items), self-esteem ( } 7 \text { items), sexual life ( } 4 \text { items), public distress ( } 5 \text { items), and } \\
\text { work ( } 4 \text { items) })^{18} \\
\text { Each item begins with the phrase "Because of my weight" and is rated on a Likert scale from } 5 \text { (always true) to } \\
1 \text { (never true) } \\
\text { Normalized IWQOL-Lite scores (separate scores for each of } 5 \text { domains and total score) range from } 0 \text { (worst } \\
\text { outcome) to } 100 \text { (best outcome) }\end{array}$ \\
\hline IW-SP & $\begin{array}{l}\text { Impact of Weight on } \\
\text { Self-Perception }\end{array}$ & $\begin{array}{l}\text { Questionnaire assessing } 3 \text { items of body weight's impact on how happy an individual is with his/her } \\
\text { appearance and public self-consciousness; rated on a 5-point numeric scale from "never" to "always"20 }\end{array}$ \\
\hline PGWB & $\begin{array}{l}\text { Psychological General } \\
\text { Well-being Index }\end{array}$ & $\begin{array}{l}\text { Brief questionnaire measuring subjective perception of well-being and distress over previous month } 21,22 \\
\text { Dimensions and score ranges include anxiety }(0-25) \text {, depressed mood }(0-15) \text {, positive well-being }(0-20) \text {, self- } \\
\text { control (0-15), general health }(0-15) \text {, and vitality (0-20); a } 20 \text { global score (sum of } 6 \text { dimensions) ranges from } \\
\text {-110; normalized scores range from 0-100, with scores for each dimension and the global score indicating } \\
\text { greater well-being22 }\end{array}$ \\
\hline SF-36 & $\begin{array}{l}36 \text {-item Short-Form } \\
\text { Survey }\end{array}$ & $\begin{array}{l}\text { 36-item questionnaire that assesses physical function, pain, general health, mental health, emotional function, } \\
\text { and social function } 23 \\
\text { Each item is scored on a scale from } 1 \text { to either } 2,3,5 \text {, or } 6 \text {; each item score is then converted to a scale of } \\
0-100 \text {, representing the percentage of total possible score achieved and with higher scores indicating a higher } \\
\text { health status; items on the same scale are then averaged to obtain the } 8 \text { scale scores (physical functioning, role } \\
\text { limitations due to physical health, role limitations due to emotional problems, energy/fatigue, emotional } \\
\text { well-being, social functioning, pain, and general health)23,24 }\end{array}$ \\
\hline \multicolumn{3}{|c|}{ Treatment satisfaction tool } \\
\hline DTSQ & $\begin{array}{l}\text { Diabetes Treatment } \\
\text { Satisfaction } \\
\text { Questionnaire }\end{array}$ & $\begin{array}{l}\text { Questionnaire measuring satisfaction with diabetes treatment consisting of } 8 \text { items evaluating } 6 \text { aspects of } \\
\text { treatment satisfaction and } 2 \text { perceived recent event rates of hyperglycemia/hypoglycemia25,26 } \\
\text { Each item is scored on a 7-point Likert scale ranging from } 0 \text { (very dissatisfied) to } 6 \text { (very satisfied) }{ }^{26} \\
\text { Items evaluating } 6 \text { aspects of treatment satisfaction are summed to produce a total treatment satisfaction score; } \\
\text { DTSQ status total scores range from } 0-36 \text {, with higher scores indicating greater satisfaction; the perceived } \\
\text { frequency of hyperglycemia/hypoglycemia items are scored separately, with lower scores indicating better } \\
\text { perceived blood glucose control26 }\end{array}$ \\
\hline
\end{tabular}

weight (based on the Impact of Weight on Self-Perceptions Questionnaire [IW-SP]), HRQoL, treatment satisfaction, and perceived hyperglycemia (Table 3). ${ }^{29,36} \mathrm{~A}$ 28-week, multicenter, randomized, double-blind study comparing dulaglutide $1.5 \mathrm{mg}$ with placebo as background therapy to insulin glargine with or without metformin (AWARD-9) found that, compared with placebo, dulaglutide was associated with significant improvements on the IW-SP and less disinhibited eating (Diabetes Health Profile [DHP-18]) but not on other patient-reported outcomes (PROs), including HRQoL (EuroQol-5D [EQ-5D]), psychological distress (DHP-18), and barriers to activity (DHP-18). ${ }^{30}$
In SUSTAIN-5, QW semaglutide $0.5 \mathrm{mg}$ and $1.0 \mathrm{mg}$, as add-on therapy to basal insulin \pm metformin, were associated with significant improvements in overall treatment satisfaction from baseline to week 30 compared with placebo (Diabetes Treatment Satisfaction Questionnaire [DTSQ]; Table 4). ${ }^{31}$

Once-Weekly GLP-1 RAs Versus Oral Antihyperglycemic Drugs A 26-week, multicenter, randomized, double-dummy study (DURATION-2) compared exenatide ER $2 \mathrm{mg}$ with maximum daily doses of sitagliptin $(100 \mathrm{mg})$ or pioglitazone $(45 \mathrm{mg})$ as add-on therapy to metformin in 491 patients with T2DM. 28,37 
Exenatide ER reduced glycated hemoglobin (Alc; -1.5\%) and weight $(-2.3 \mathrm{~kg})$ to a significantly $(P<0.05)$ greater degree than pioglitazone $(-1.2 \%,+2.8 \mathrm{~kg})$ and sitagliptin $(-0.9 \%$, $-0.8 \mathrm{~kg}$ ); no episodes of major hypoglycemia were reported. ${ }^{37}$ Weight-related HRQoL as measured by the Impact of Weight on Quality of Life-Lite (IWQOL-Lite) questionnaire improved significantly from baseline in patients receiving exenatide ER or sitagliptin but not pioglitazone (Table 2). ${ }^{28}$

Improvement in weight-related HRQoL (IWQoL-Lite) was significantly greater with exenatide versus pioglitazone. Similarly, general health utility scores (EQ-5D) improved significantly for patients receiving exenatide ER or sitagliptin but not pioglitazone, although there were no significant differences between treatment groups. All treatment groups demonstrated significant improvements in psychological well-being (Psychological General Well-being Index [PGWB]) and diabetes treatment satisfaction (DTSQ), with no significant differences between exenatide ER and either comparator. Across the entire study population, the development of nausea/vomiting did not affect treatment satisfaction. However, reduction in body weight was found to correlate significantly with improvements in overall weight-related HRQoL $(r=-0.26, P<0.0001)$.

AWARD-3 was a 52-week, multicenter, randomized, doubleblind, double-dummy study comparing dulaglutide $0.75 \mathrm{mg}$, dulaglutide $1.5 \mathrm{mg}$, and metformin in patients with T2DM managed with diet and exercise. ${ }^{29,36,38}$ Both doses of dulaglutide $(-0.7 \%$ [dulaglutide $0.75 \mathrm{mg}$ ], $-0.8 \%$ [dulaglutide $1.5 \mathrm{mg}$ ]) produced significantly greater reductions in Alc than metformin $(-0.6 \%)$ at 26 weeks, whereas mean weight reduction at 26 weeks was similar between dulaglutide $1.5 \mathrm{mg}(-2.3 \mathrm{~kg})$ and metformin $(-2.2 \mathrm{~kg})$ but was less with dulaglutide $0.75 \mathrm{mg}$ $(-1.4 \mathrm{~kg})$. No severe hypoglycemic events occurred. At week 26, there were no significant differences between dulaglutide and metformin with regard to improvements in impact of weight on self-perception (IW-SP), Ability to Perform Physical Activities of Daily Living scale (APPADL), or treatment satisfaction, with the exception of significantly $(P<0.05)$ greater improvement in perceived hyperglycemia (DTSQs-hyperglycemia score) in the dulaglutide 1.5 QW group (Table 3). ${ }^{29,36}$ The latter finding is consistent with the greater reductions in Alc observed with dulaglutide compared with metformin. Although there was a statistically significant relationship between change in Alc and change in treatment satisfaction, the strength of the relationship was of questionable clinical significance (slope estimate for regression analysis, $-1.058 ; P \leq 0.001) .{ }^{36}$ There was no significant relationship between weight or hypoglycemia incidence and change in treatment satisfaction.

In AWARD-5, a 104-week, multicenter, randomized, doubleblind, placebo-controlled study with a primary endpoint of 52 weeks, dulaglutide $0.75 \mathrm{mg}$ and $1.5 \mathrm{mg}$ were compared with sitagliptin in patients with T2DM on background metformin therapy. ${ }^{39}$ Placebo-treated patients were switched to sitagliptin after 26 weeks. At 52 weeks, both dulaglutide doses were associated with significantly greater reductions in Alc (-0.9\% [dulaglutide $0.75 \mathrm{mg}$ ], $-1.1 \%$ [dulaglutide $1.5 \mathrm{mg}$ ]) and weight (2.6 kg [dulaglutide $0.75 \mathrm{mg}$ ], $-3.0 \mathrm{~kg}$ [dulaglutide $1.5 \mathrm{mg}$ ]) compared with sitagliptin (Alc: $-0.4 \%$; body weight: $-1.5 \mathrm{~kg}$ ). No severe hypoglycemic events occurred. However, there were no significant differences at 52 weeks between either dose of dulaglutide and sitagliptin on measures of HRQoL and impact of weight on quality of life (Table 3). ${ }^{29}$

SUSTAIN-2 was a 56-week, multicenter, randomized, double-blind, double-dummy, placebo-controlled study that compared semaglutide $0.5 \mathrm{mg}$ and $1.0 \mathrm{mg}$ with sitagliptin as add-on therapy to metformin, a thiazolidinedione, or a combination of metformin with a thiazolidinedione in patients with T2DM. ${ }^{33}$ Compared with sitagliptin, both doses of semaglutide produced significantly greater reductions from baseline to week 56 in Alc (-1.3\% [semaglutide $0.5 \mathrm{mg}$ ], -1.6\% [semaglutide $1.0 \mathrm{mg}$ ], $-0.5 \%$ [sitagliptin]) and body weight $(-4.3 \mathrm{~kg}$ [semaglutide $0.5 \mathrm{mg}$ ], $-6.1 \mathrm{~kg}$ [semaglutide $1.0 \mathrm{mg}$ ], $-1.9 \mathrm{~kg}$ [sitagliptin]). Severe hypoglycemia occurred in no semaglutidetreated patients and in 2 patients receiving sitagliptin. Overall treatment satisfaction and perceived hyperglycemia (DTSQ) improved to a significantly greater extent in the semaglutide groups than in the sitagliptin group (Table 4). Compared with patients taking sitagliptin, responses to individual DTSQ items indicated that those taking semaglutide found their treatment to be more convenient (1.0 mg dose) and more flexible (both doses), were more satisfied with their understanding of their diabetes (1.0 mg dose), were more willing to continue their current treatment (1.0 mg dose), and were more likely to recommend their treatment (both doses). Further, several aspects of HRQoL, as measured on the 36-item Short-Form Survey (SF-36), improved with semaglutide versus sitagliptin (Table 4).

\section{Once-Weekly GLP-1 RAs Versus Insulin}

Dulaglutide (0.75 and $1.5 \mathrm{mg}$ ) was compared with insulin glargine at week 26 (primary endpoint) as add-on therapy to insulin lispro with or without metformin (AWARD-4) ${ }^{40}$ and at week 52 (primary endpoint) in patients receiving metformin and glimepiride (AWARD-2). ${ }^{41}$ In AWARD-4, both doses of dulaglutide were associated with significantly greater reductions in Alc than insulin glargine at week $26(-1.6 \%$ [dulaglutide $0.75 \mathrm{mg}$ ], $-1.6 \%$ [dulaglutide $1.5 \mathrm{mg}$ ], $-1.4 \%$ [insulin glargine]). ${ }^{40}$ Body weight decreased with dulaglutide $1.5 \mathrm{mg}(-0.9 \mathrm{~kg})$ and increased slightly with dulaglutide $0.75 \mathrm{mg}(+0.2 \mathrm{~kg})$, but both doses were significantly different versus insulin glargine $(+2.3 \mathrm{~kg}$; i.e., greater weight gain was observed with insulin). Compared with insulin glargine, incidence rates of hypoglycemia (plasma glucose $\leq 3.9 \mathrm{mmol} / \mathrm{L}$ ) at week 26 were similar with both doses of dulaglutide $(88.4 \%$ [dulaglutide $0.75 \mathrm{mg}$ ], $85.9 \%$ [dulaglutide 1.5 $\mathrm{mg}$ ], 89.5\% [insulin glargine]). At week 26, the only significant difference between dulaglutide and insulin glargine in PROs 
Health-Related Quality of Life Assessments with Once-Weekly

Glucagon-Like Peptide-1 Receptor Agonists in Type 2 Diabetes Mellitus

TABLE 2 Changes from Baseline in Patient-Reported Outcomes in the DURATION Clinical Trial Program with Once-Weekly Exenatide ER Compared with Other Antidiabetic Therapies

\begin{tabular}{|c|c|c|c|c|c|c|c|c|}
\hline \multirow[b]{2}{*}{ Variable } & \multicolumn{4}{|c|}{ DURATION-1 (Week 30)27 } & \multicolumn{4}{|c|}{ DURATION-1 (Week 30-52) ${ }^{27}$} \\
\hline & \multicolumn{2}{|c|}{$\begin{array}{c}\begin{array}{c}\text { Exenatide ER } 2 \mathrm{mg} \\
(\mathrm{n}=148)\end{array} \\
\text { LSM Change (SE) }\end{array}$} & \multicolumn{2}{|c|}{$\begin{array}{c}\text { Exenatide BID } 10 \mu \mathrm{g} \\
(\mathrm{n}=147) \\
\text { LSM Change (SE) }\end{array}$} & \multicolumn{2}{|c|}{$\begin{array}{c}\text { Exenatide ER } 2 \text { mg } \\
\text { LSM Change (SE) }\end{array}$} & \multicolumn{2}{|c|}{$\begin{array}{c}\text { Exenatide } 10 \mu \mathrm{g} \\
\text { BID } \rightarrow \text { Exenatide ER } 2 \mathrm{mg} \\
\text { LSM Change (SE) }\end{array}$} \\
\hline \multicolumn{9}{|l|}{$\overline{\text { DTSQ }}$} \\
\hline DTSQs total score & 5.17 & $(0.54)^{\mathrm{a}}$ & 3.97 & $(0.53)^{\mathrm{a}}$ & 0.65 & $(3.8)$ & 1.16 & $(6.1)^{\mathrm{b}}$ \\
\hline Treatment satisfaction-current & 1.29 & $(0.12)^{a}$ & 1.11 & $(0.11)^{\mathrm{a}}$ & 0.10 & $(0.77)$ & 0.01 & $(1.2)$ \\
\hline Perceived frequency hyperglycemia & -1.86 & $(0.15)^{a, c}$ & -1.42 & $(0.15)^{\mathrm{a}}$ & -0.04 & $(1.9)$ & -0.15 & $(1.8)$ \\
\hline Perceived frequency hypoglycemia & 0.08 & $(0.12)$ & 0.14 & $(0.11)$ & -0.18 & $(1.4)$ & -0.21 & $(1.4)$ \\
\hline Treatment convenience & 0.25 & $(0.13)^{\mathrm{d}}$ & 0.14 & $(0.13)$ & 0.30 & $(1.2)^{\mathrm{e}}$ & 0.42 & $(1.6)^{\mathrm{e}}$ \\
\hline Treatment flexibility & 0.49 & $(0.13)^{\mathrm{a}}$ & 0.27 & $(0.13)^{\mathrm{d}}$ & 0.24 & $(1.2)^{b}$ & 0.39 & $(1.7)^{\mathrm{b}}$ \\
\hline Understanding of diabetes & 0.50 & $(0.10)^{\mathrm{a}}$ & 0.56 & $(0.10)^{\mathrm{a}}$ & 0.06 & $(0.94)$ & 0.04 & $(1.2)$ \\
\hline Treatment recommend & 0.98 & $(0.10)^{\mathrm{a}}$ & 0.83 & $(0.10)^{\mathrm{a}}$ & -0.05 & $(0.94)$ & 0.07 & $(1.1)$ \\
\hline Treatment satisfaction-continue & 1.43 & $(0.13)^{\mathrm{a}, \mathrm{c}}$ & 0.99 & $(0.12)^{\mathrm{a}}$ & -0.01 & $(0.93)$ & 0.24 & $(1.3)^{\mathrm{b}}$ \\
\hline \multicolumn{9}{|l|}{ IWQOL-Lite } \\
\hline IWQOL-Lite total score & 10.23 & $(0.89)^{a}$ & 8.61 & $(0.88)^{\mathrm{a}}$ & 0.36 & $(7.1)$ & 1.44 & $(8.7)$ \\
\hline Physical function & 12.06 & $(1.20)^{\mathrm{a}}$ & 9.55 & $(1.21)^{\mathrm{a}}$ & 0.08 & $(9.5)$ & 2.13 & $(11.5)^{b}$ \\
\hline Self-esteem & 13.60 & $(1.31)^{\mathrm{a}}$ & 13.71 & $(1.30)^{\mathrm{a}}$ & 0.83 & $(10.9)$ & 1.12 & $(11.5)$ \\
\hline Sexual life & 7.34 & $(1.51)^{\mathrm{a}}$ & 6.95 & $(1.50)^{\mathrm{a}}$ & 0.64 & $(17.3)$ & 0.91 & $(15.2)$ \\
\hline Public distress & 6.05 & $(1.00)^{\mathrm{a}}$ & 4.07 & $(1.00)^{\mathrm{a}}$ & 6.96 & $(13.2)^{f}$ & 5.04 & $(11.2)^{\mathrm{f}}$ \\
\hline \multirow[t]{3}{*}{ Work } & 8.17 & $(1.13)^{\mathrm{a}}$ & 5.81 & $(1.12)^{\mathrm{a}}$ & 0.42 & $(9.8)$ & 1.10 & $(11.2)$ \\
\hline & \multicolumn{6}{|c|}{ DURATION-2 (Week 26) 28} & & \\
\hline & Exenatide ER & $2 \mathrm{mg}(\mathrm{n}=160)$ & Sitaglipti & $(n=166)$ & Pioglitaze & ne $(n=165)$ & & \\
\hline Variable & LSM Chr & nge (SE) & LSM Ch & nge (SE) & LSM Ch & ange (SE) & & \\
\hline \multicolumn{7}{|l|}{ DTSQ } & & \\
\hline Total score & 3.96 & $(0.60)^{\mathrm{d}}$ & 2.35 & $(0.59)^{\mathrm{d}}$ & 2.50 & $(0.61)^{d}$ & & \\
\hline Perceived frequency hyperglycemia & -1.63 & $(0.17)^{\mathrm{d}}$ & -1.30 & $(0.17)^{\mathrm{d}}$ & -1.28 & $(0.17)^{\mathrm{d}}$ & & \\
\hline Perceived frequency hypoglycemia & 0.22 & $(0.15)$ & -0.05 & $(0.15)$ & -0.12 & $(0.15)$ & & \\
\hline \multicolumn{7}{|l|}{ IWQOL-Lite } & & \\
\hline IWQOL-Lite total score & 5.15 & $(1.04)^{\mathrm{d}}$ & 4.56 & $(1.02)^{\mathrm{d}}$ & 1.20 & $(1.06)^{g}$ & & \\
\hline Physical function & 6.78 & $(1.35)^{\mathrm{d}}$ & 5.81 & $(1.33)^{\mathrm{d}}$ & 2.00 & $(1.38) \mathrm{g}$ & & \\
\hline Self-esteem & 5.88 & $(1.39)^{\mathrm{d}}$ & 5.79 & $(1.36)^{\mathrm{d}}$ & 3.11 & $(1.41)$ & & \\
\hline Sexual life & 5.80 & $(1.61) \mathrm{d}$ & 5.02 & $(1.61) \mathrm{d}$ & 2.41 & $(1.63)$ & & \\
\hline Public distress & 3.86 & $(1.17)^{\mathrm{d}}$ & 2.40 & $(1.14)^{\mathrm{d}}$ & -0.63 & $(1.18)^{g}$ & & \\
\hline Work & 2.79 & $(1.28)^{\mathrm{d}}$ & 3.02 & $(1.25)^{\mathrm{d}}$ & -1.28 & $(1.29) \mathrm{g}$ & & \\
\hline \multicolumn{7}{|l|}{ EQ-5D } & & \\
\hline Index score & 0.04 & $(0.02)^{\mathrm{d}}$ & 0.05 & $(0.02)^{\mathrm{d}}$ & 0.02 & $(0.02)$ & & \\
\hline Visual analog scale score & 4.46 & $(1.34)^{\mathrm{d}}$ & 6.04 & $(1.32)^{\mathrm{d}}$ & 2.54 & $(1.37)$ & & \\
\hline \multicolumn{7}{|l|}{ PGWB } & & \\
\hline Global score & 6.82 & $(1.00)^{\mathrm{d}}$ & 6.97 & $(0.98)^{\mathrm{d}}$ & 4.78 & $(1.02)^{\mathrm{d}}$ & & \\
\hline Anxiety & 8.40 & $(1.31)^{\mathrm{d}}$ & 8.20 & $(1.28)^{\mathrm{d}}$ & 5.10 & $(1.33)^{\mathrm{d}}$ & & \\
\hline Depressed mood & 3.84 & $(1.33)^{\mathrm{d}}$ & 3.80 & $(1.30)^{\mathrm{d}}$ & 3.73 & $(1.36)^{\mathrm{d}}$ & & \\
\hline Positive well-being & 4.65 & $(1.42)^{\mathrm{d}}$ & 7.86 & $(1.39)^{\mathrm{d}}$ & 5.02 & $(1.45)^{\mathrm{d}}$ & & \\
\hline Self-control & 5.53 & $(1.37)^{\mathrm{d}}$ & 4.30 & $(1.34)^{\mathrm{d}}$ & 3.68 & $(1.40)^{\mathrm{d}}$ & & \\
\hline General health & 9.46 & $(1.40)^{\mathrm{d}}$ & 6.95 & $(1.37)^{\mathrm{d}}$ & 6.37 & $(1.43) \mathrm{d}$ & & \\
\hline Vitality & 7.46 & $(1.37)^{\mathrm{d}}$ & 8.98 & $(1.35)^{\mathrm{d}}$ & 6.23 & $(1.41)^{\mathrm{d}}$ & & \\
\hline
\end{tabular}

aP $<0.001$ from baseline to week 30 .

${ }^{b} P \leq 0.05$ from week 30 to week 52 .

$c P<0.05$ exenatide ER versus exenatide BID.

${ }^{d} P \leq 0.05$ versus baseline to week $30 / 26$.

${ }^{e} \mathrm{P}<0.01$ from week 30 to week 52 .

${ }^{f} P<0.001$ from week 30 to week 52 .

$g P \leq 0.05$ versus exenatide $E R$.

$B I D=$ twice weekly; DTSQ = Diabetes Treatment Satisfaction Questionnaire; DTSQs=Diabetes Treatment Satisfaction Questionnaire-Status version; EQ-5D=EuroQol 5 Dimensions; ER=extended release; LSM=least squares mean; IWQOL-Lite=Impact of Weight on Quality of Life-Lite; PGWB=Psychological General Well-Being Index; $S E=$ standard error. 
Health-Related Quality of Life Assessments with Once-Weekly

Glucagon-Like Peptide-1 Receptor Agonists in Type 2 Diabetes Mellitus

TABLE 3 Changes from Baseline in Patient-Reported Outcomes in the AWARD Clinical Trial Program with Once-Weekly Dulaglutide Compared with Other Antidiabetic Therapies ${ }^{29}$

\begin{tabular}{|c|c|c|c|c|c|c|}
\hline \multirow[b]{2}{*}{ Variable } & \multicolumn{3}{|c|}{ AWARD-3 (Week 26) } & \multicolumn{3}{|c|}{ AWARD-5 (Week 52) } \\
\hline & $\begin{array}{c}\text { Dulaglutide } \\
1.5 \mathrm{mg}(\mathrm{n}=269)\end{array}$ & $\begin{array}{c}\text { Dulaglutide } \\
0.75 \mathrm{mg}(\mathrm{n}=270)\end{array}$ & $\begin{array}{l}\text { Metformin } \\
(\mathrm{n}=268)\end{array}$ & $\begin{array}{c}\text { Dulaglutide } \\
1.5 \mathrm{mg}(\mathrm{n}=279)\end{array}$ & $\begin{array}{c}\text { Dulaglutide } \\
0.75 \mathrm{mg}(\mathrm{n}=280)\end{array}$ & $\begin{array}{l}\text { Sitagliptin } \\
(\mathrm{n}=276)\end{array}$ \\
\hline IW-SPa & $6.0(1.6)^{b}$ & $5.3(1.5)^{\mathrm{b}}$ & $6.6(1.5)^{\mathrm{b}}$ & - & - & - \\
\hline APPADL $^{a}$ & $0.3 \quad(1.2)$ & $0.7 \quad(1.2)$ & $0.1 \quad(1.2)$ & - & - & - \\
\hline IWQOL-Lite $^{\mathrm{a}}$ & - & - & - & $3.3(0.7)^{\mathrm{b}}$ & $2.7(0.7)^{\mathrm{b}}$ & $2.1(0.7)^{\mathrm{b}}$ \\
\hline EQ-5D UK Index & - & - & - & $0.00(0.01)$ & $0.01(0.01)$ & $0.00(0.01)$ \\
\hline EQ-5D VAS & - & - & - & $2.1 \quad(0.8)^{b}$ & $1.5 \quad(0.8)$ & $1.2(0.8)$ \\
\hline $\mathrm{DTSQ}_{S^{\mathrm{c}}}$ & $1.9(0.4)^{\mathrm{b}}$ & $1.8(0.4)^{\mathrm{b}}$ & $2.0 \quad(0.4)^{b}$ & - & - & - \\
\hline DTSQs-Hyperglycemia & $-1.3(0.1)^{\mathrm{b}, \mathrm{d}}$ & $-1.1 \quad(0.1)^{\mathrm{b}}$ & $-0.9(0.1)^{b}$ & - & - & - \\
\hline DTSQs-Hypoglycemia & $-0.1 \quad(0.1)$ & $-0.1 \quad(0.1)$ & $0.1 \quad(0.1)$ & - & - & - \\
\hline \multirow[t]{3}{*}{ DSC-R } & $0.2 \quad(0.4)$ & $-0.2(0.4)$ & $0.4 \quad(0.4)$ & - & - & - \\
\hline & \multicolumn{3}{|c|}{ AWARD-2 (Week 52) } & \multicolumn{3}{|c|}{ AWARD-4 (Week 26) } \\
\hline & $\begin{array}{c}\text { Dulaglutide } \\
1.5 \mathrm{mg}(\mathrm{n}=273)\end{array}$ & $\begin{array}{c}\text { Dulaglutide } \\
0.75 \mathrm{mg}(\mathrm{n}=272)\end{array}$ & $\begin{array}{l}\text { Insulin glargine } \\
\quad(\mathrm{n}=262)\end{array}$ & $\begin{array}{c}\text { Dulaglutide } \\
1.5 \mathrm{mg}(\mathrm{n}=295)\end{array}$ & \begin{tabular}{|c|} 
Dulaglutide \\
$0.75 \mathrm{mg}(\mathrm{n}=293)$
\end{tabular} & $\begin{array}{c}\text { Insulin glargine } \\
(\mathbf{n}=296)\end{array}$ \\
\hline IW-SPa & $3.8(1.3)^{\mathrm{b}}$ & $2.0 \quad(1.3)$ & $1.2 \quad(1.4)$ & $4.9 \quad(1.4)^{\mathrm{b}, \mathrm{d}}$ & $3.0(1.4)^{b}$ & $1.5 \quad(1.4)$ \\
\hline APPADL $^{a}$ & $3.4 \quad(1.1)^{\mathrm{b}, \mathrm{d}}$ & $1.4 \quad(1.1)^{\mathrm{d}}$ & $-2.1 \quad(1.1)$ & $-1.8 \quad(1.1)$ & $-2.1 \quad(1.1)$ & $-3.1(1.1)^{b}$ \\
\hline EQ-5D UK Index & $0.01(0.01)^{\mathrm{d}}$ & $0.00(0.01)^{\mathrm{d}}$ & $-0.04(0.01)^{b}$ & $-0.03(0.01)^{b}$ & $-0.02(0.01)$ & $-0.03(0.01)^{b}$ \\
\hline EQ-5D VAS & $3.2(0.9)^{\mathrm{b}}$ & $2.3(0.9)^{\mathrm{b}}$ & $1.1 \quad(0.9)$ & $-1.4 \quad(1.0)$ & $-1.0 \quad(1.0)$ & $-0.4 \quad(1.0)$ \\
\hline ALBSSC & $-4.2(0.8)^{\mathrm{b}, \mathrm{d}}$ & $-4.1 \quad(0.8)^{\mathrm{b}, \mathrm{d}}$ & $-1.0 \quad(0.9)$ & $3.5(1.3)^{\mathrm{b}}$ & $3.3(1.3)^{\mathrm{b}}$ & $3.3(1.3)^{\mathrm{b}}$ \\
\hline ALBSS-Worry & $-2.2(0.5)^{b, d}$ & $-2.1(0.5)^{\mathrm{b}, \mathrm{d}}$ & $-0.3(0.5)$ & $2.1 \quad(0.9)^{\mathrm{b}}$ & $1.1 \quad(0.9)$ & $2.1 \quad(0.9)^{\mathrm{b}}$ \\
\hline \multirow[t]{3}{*}{ ALBSS-Behavior } & $-2.1(0.5)^{\mathrm{b}, \mathrm{d}}$ & $-2.1(0.5)^{\mathrm{b}, \mathrm{d}}$ & $-0.8 \quad(0.5)$ & $1.3(0.6)^{\mathrm{b}}$ & $2.2(0.6)^{\mathrm{b}}$ & $1.2(0.6)^{b}$ \\
\hline & \multicolumn{4}{|c|}{ AWARD-1 (Week 26) } & \multicolumn{2}{|c|}{ AWARD-6 (Week 26) } \\
\hline & $\begin{array}{c}\text { Dulaglutide } \\
1.5 \mathrm{mg}(\mathrm{n}=279)\end{array}$ & $\begin{array}{c}\text { Dulaglutide } \\
0.75 \mathrm{mg}(\mathrm{n}=280)\end{array}$ & $\begin{array}{c}\text { Exenatide } \\
(\mathbf{n}=276)\end{array}$ & $\begin{array}{l}\text { Placebo } \\
(\mathrm{n}=141)\end{array}$ & $\begin{array}{c}\text { Dulaglutide } \\
1.5 \mathrm{mg}(\mathrm{n}=299)\end{array}$ & $\begin{array}{c}\text { Liraglutide } \\
1.8 \mathrm{mg}(\mathrm{n}=300)\end{array}$ \\
\hline IW-SPa & $4.7 \quad(1.2)^{\mathrm{b}}$ & $3.9(1.2)^{\mathrm{b}}$ & $3.9(1.2)^{b}$ & $3.7(1.6)^{b}$ & $7.4(1.3)^{\mathrm{b}}$ & $7.7(1.3)^{\mathrm{b}}$ \\
\hline APPADL $^{a}$ & $0.6 \quad(1.0)$ & $0.4 \quad(1.0)$ & $1.7 \quad(1.0)$ & $0.1 \quad(1.3)$ & $2.6(1.1)^{\mathrm{b}}$ & $0.5 \quad(1.1)$ \\
\hline EQ-5D UK Index & $0.01(0.01)$ & $0.01(0.01)$ & $0.00(0.01)$ & $0.00(0.02)$ & $0.02(0.01)^{b}$ & $0.01(0.01)$ \\
\hline EQ-5D VAS & $4.5(0.9)^{\mathrm{b}, \mathrm{e}}$ & $2.4(0.9)^{\mathrm{b}}$ & $3.9 \quad(0.9)^{\mathrm{b}, \mathrm{e}}$ & $0.7 \quad(1.2)$ & $2.8(0.8)^{\mathrm{b}}$ & $1.8(0.8)^{\mathrm{b}}$ \\
\hline DTSQs $^{c}$ & $2.4 \quad(0.3)^{b, d, e}$ & $2.6(0.3)^{\mathrm{b}, \mathrm{d}, \mathrm{e}}$ & $0.9(0.3)^{b}$ & $0.5 \quad(0.5)$ & - & - \\
\hline DTSQs-Hyperglycemia & $-1.9(0.1)^{\mathrm{b}, \mathrm{d}, \mathrm{e}}$ & $-1.8(0.1)^{\mathrm{b}, \mathrm{d}, \mathrm{e}}$ & $-1.1 \quad(0.1)^{\mathrm{b}, \mathrm{e}}$ & $-0.5(0.2)^{b}$ & - & - \\
\hline \multirow[t]{3}{*}{ DTSQs-Hypoglycemia } & $0.1 \quad(0.1)^{\mathrm{d}}$ & $0.2(0.1)^{\mathrm{d}}$ & $0.5(0.1)^{\mathrm{b}}$ & $0.3 \quad(0.2)$ & - & - \\
\hline & \multicolumn{2}{|c|}{ AWARD-9 (Week 28) } & & & & \\
\hline & $\begin{array}{c}\text { Dulaglutide } \\
1.5 \mathrm{mg}(\mathrm{n}=150)\end{array}$ & $\begin{array}{l}\text { Placebo } \\
(\mathrm{n}=150)\end{array}$ & & & & \\
\hline IW-SPa & $6.14(2.25)^{\mathrm{b}, \mathrm{e}}$ & $0.07(2.20)$ & & & & \\
\hline EQ-5D UK Index & $-0.03(0.02)$ & $-0.02(0.02)$ & & & & \\
\hline EQ-5D VAS & $2.73(1.37)^{\mathrm{e}}$ & $0.65(1.33)$ & & & & \\
\hline DHP-18 (psychological distress domain) & $0.13(1.39)$ & $0.89(1.35)$ & & & & \\
\hline DHP-18 (barriers to activity domain) & $-0.63(1.48)$ & $2.21(1.43)$ & & & & \\
\hline DHP-18 (disinhibited eating domain) & $-5.69(1.70)^{\mathrm{b}, \mathrm{e}}$ & $-1.18(1.65)$ & & & & \\
\hline \multicolumn{7}{|c|}{$\begin{array}{l}\text { Note: Data are presented as least squares mean (standard error). } \\
\text { aTotal score transformed. } \\
{ }^{b} P<0.05 \text { versus placebo. } \\
\text { 'Total score. } \\
{ }^{2} P<0.05 \text { versus active comparator. } \\
\text { eP } P 0.05 \text { versus baseline. } \\
\text { ALBSS = Adult Low Blood Sugar Survey; APPADL=Ability to Perform Physical Activities of Daily Living; DTSQs = Diabetes Treatment Satisfaction Questionnaire-Status } \\
\text { version; DSC-R = Diabetes Symptom Checklist-Revised; EQ-5D=EuroQol } 5 \text { Dimensions; IWQOL-Lite=Impact of Weight on Quality of Life-Lite; IW-SP=Impact of } \\
\text { Weight on Self-Perception; VAS = visual analog scale. }\end{array}$} \\
\hline
\end{tabular}


TABLE 4 Changes from Baseline in Patient-Reported Outcomes in the SUSTAIN Clinical Trial Program with Once-Weekly Semaglutide Compared with Other Antidiabetic Therapies

\begin{tabular}{|c|c|c|c|c|c|c|c|c|}
\hline \multirow[b]{3}{*}{ Variable } & \multicolumn{5}{|c|}{ SUSTAIN-5 (30 Weeks) $)^{31}$} & \multicolumn{3}{|c|}{ SUSTAIN-3 (56 Weeks) ${ }^{32}$} \\
\hline & \multirow{2}{*}{$\begin{array}{c}\text { Semaglutide } \\
0.5 \mathrm{mg}(\mathrm{n}=132) \\
\text { Mean Change }\end{array}$} & \multirow{2}{*}{\multicolumn{2}{|c|}{$\begin{array}{c}\text { Semaglutide } \\
1.0 \mathrm{mg}(\mathrm{n}=131) \\
\text { Mean Change }\end{array}$}} & \multicolumn{2}{|c|}{$\begin{array}{l}\text { Placebo } \\
(\mathrm{n}=133)\end{array}$} & \multicolumn{2}{|c|}{$\begin{array}{c}\text { Semaglutide } \\
1.0 \mathrm{mg}(\mathrm{n}=404)\end{array}$} & $\begin{array}{c}\text { Exenatide ER } \\
2.0 \mathrm{mg}(\mathrm{n}=405)\end{array}$ \\
\hline & & & & \multicolumn{2}{|c|}{ Mean Change } & \multicolumn{2}{|c|}{ Mean Change } & Mean Change \\
\hline \multirow[t]{3}{*}{ DTSQs } & $2.7^{\mathrm{a}}$ & & $3.5^{\mathrm{b}}$ & & & $5.0^{\mathrm{b}}$ & & 4.0 \\
\hline & \multicolumn{4}{|c|}{ SUSTAIN-2 (56 Weeks) ${ }^{33}$} & \multicolumn{4}{|c|}{ SUSTAIN-4 $\left(30\right.$ Weeks) ${ }^{34}$} \\
\hline & \multirow{2}{*}{\multicolumn{2}{|c|}{$\begin{array}{c}\text { Semaglutide } \\
0.5 \mathrm{mg}(\mathrm{n}=409) \\
\text { Mean }(95 \% \mathrm{CI}) \text { ETD } \\
\text { vs. Sitagliptin }\end{array}$}} & \multirow{2}{*}{\multicolumn{2}{|c|}{$\begin{array}{c}\text { Semaglutide } \\
1.0 \mathrm{mg}(\mathrm{n}=409) \\
\text { Mean }(95 \% \mathrm{CI}) \text { ETD } \\
\text { vs. Sitagliptin }\end{array}$}} & \multirow{2}{*}{\multicolumn{2}{|c|}{$\begin{array}{c}\text { Semaglutide } \\
0.5 \mathrm{mg}(\mathrm{n}=362) \\
\text { Mean }(95 \% \mathrm{CI}) \text { ETD } \\
\text { vs. Insulin Glargine }\end{array}$}} & \multirow{2}{*}{\multicolumn{2}{|c|}{$\begin{array}{c}\text { Semaglutide } \\
1.0 \mathrm{mg}(\mathrm{n}=360) \\
\text { Mean }(95 \% \mathrm{CI}) \text { ETD } \\
\text { vs. Insulin Glargine }\end{array}$}} \\
\hline Variable & & & & & & & & \\
\hline \multicolumn{9}{|l|}{ DTSQ } \\
\hline DTSQs total score & $(0.18,1$. & & 1.46 & $1,2.11)^{\mathrm{a}}$ & 0.87 & $(0.11,1.63)^{a}$ & 1.38 & $(0.60,2.15)^{\mathrm{a}}$ \\
\hline Treatment satisfaction-current & $(0.01,0$. & & 0.28 & $4,0.42)^{\mathrm{a}}$ & 0.03 & $(-0.16,0.21)$ & 0.16 & $(-0.03,0.34)$ \\
\hline Perceived frequency hyperglycemia & $-0.38 \quad(-0.64,-0$ & & $-0.61 \quad(-0$ & $7,-0.35)^{\mathrm{a}}$ & -0.44 & $(-0.70,-0.17)^{\mathrm{a}}$ & -0.71 & $(-0.97,-0.44)^{a}$ \\
\hline Perceived frequency hypoglycemia & $0.08 \quad(-0.13,0$. & & $-0.02 \quad(-0$. & $2,0.19)$ & -0.02 & $(-0.25,0.20)$ & -0.07 & $(-0.30,0.16)$ \\
\hline Treatment convenience & $0.13(-0.02,0$. & & 0.21 & $5,0.37)^{\mathrm{a}}$ & 0.20 & $(0.03,0.37)^{\mathrm{a}}$ & 0.17 & $(-0.00,0.34)$ \\
\hline Treatment flexibility & $0.18 \quad(0.02,0$. & & 0.19 & $4,0.35)^{\mathrm{a}}$ & 0.16 & $(-0.01,0.34)$ & 0.33 & $(0.15,0.51)^{\mathrm{a}}$ \\
\hline Understanding of diabetes & $0.02 \quad(-0.14,0$. & & 0.16 & $1,0.32)^{a}$ & 0.04 & $(-0.11,0.19)$ & 0.07 & $(-0.09,0.22)$ \\
\hline Treatment recommendation & $(0.07,0$. & & 0.31 & $7,0.44)^{\mathrm{a}}$ & 0.14 & $(-0.02,0.30)$ & 0.24 & $(0.08,0.40)^{\mathrm{a}}$ \\
\hline Treatment satisfaction-continue & $0.10 \quad(-0.04,0$. & & 0.25 & $1,0.39)^{\mathrm{a}}$ & 0.28 & $(0.09,0.47)^{\mathrm{a}}$ & 0.36 & $(0.17,0.56)^{\mathrm{a}}$ \\
\hline \multicolumn{9}{|l|}{ SF-36 } \\
\hline Physical component summary & $-0.11 \quad(-1.04,0$. & & $0.21 \quad(-0$ & $1,1.14)$ & 0.00 & $(-0.97,0.98)$ & 0.91 & $(-0.07,1.89)$ \\
\hline Mental component summary & $1.72 \quad(0.54,2$ & & $1.64 \quad(0$ & $7,2.81)^{\mathrm{a}}$ & 0.98 & $(-0.28,2.25)$ & 1.08 & $(-0.20,2.35)$ \\
\hline Physical functioning & $0.10 \quad(-0.88,1$. & & $0.44 \quad(-0$ & $4,1.42)$ & 0.96 & $(-0.21,2.12)$ & 0.08 & $(-0.37,1.97)$ \\
\hline Role physical & $0.30 \quad(-0.80,1$. & & $0.92 \quad(-0$ & $7,2.02)$ & 0.12 & $(-1.12,1.36)$ & 1.19 & $(-0.06,2.44)$ \\
\hline Bodily pain & $0.71 \quad(-0.68,2$ & & $-0.13 \quad(-1$ & $1,1.25)$ & 0.05 & $(-1.34,1.43)$ & 0.86 & $(-0.53,2.25)$ \\
\hline General health & $0.40 \quad(-0.65,1$. & & $0.96 \quad(-0$ & $9,2.01)$ & 0.33 & $(-0.70,1.35)$ & 1.15 & $(0.12,2.18)^{a}$ \\
\hline Vitality & $(0.36,2$. & & 1.31 & $5,2.47)^{\mathrm{a}}$ & 0.75 & $(-0.49,2.00)$ & 1.13 & $(-0.12,2.38)$ \\
\hline Social functioning & $0.62(-0.53,1$. & & $1.06 \quad(-0$. & $9,2.21)$ & 0.77 & $(-0.52,2.06)$ & 0.68 & $(-0.62,1.98)$ \\
\hline Role emotional & $0.81 \quad(-0.46,2$ & & $1.43 \quad(0$ & $7,2.70)^{\mathrm{a}}$ & 0.83 & $(-0.63,2.29)$ & 1.67 & $(0.20,3.14)^{\mathrm{a}}$ \\
\hline Mental health & $2.03 \quad(0.78,3$. & & $1.24 \quad(-0$. & $0,2.49)$ & 1.15 & $(-0.11,2.40)$ & 0.62 & $(-0.64,1.89)$ \\
\hline
\end{tabular}

continued on next page

was significantly greater improvement in impact of weight on self-perception (IW-SP) with dulaglutide $1.5 \mathrm{mg}$ (Table 3). ${ }^{29,40} \mathrm{In}$ AWARD-2, the higher dulaglutide dose (-1.1\%) produced a significantly greater reduction in Alc than insulin glargine (-0.6\%) at week $52 .{ }^{41}$ Both doses of dulaglutide were associated with weight loss (-1.3 kg [dulaglutide $0.75 \mathrm{mg}$ ], $-1.9 \mathrm{~kg}$ [dulaglutide $1.5 \mathrm{mg}$ ]), while insulin glargine was associated with weight gain $(1.4 \mathrm{~kg})$; the difference versus insulin glargine was significant for both dulaglutide doses. Incidence rates of total hypoglycemia were significantly lower for dulaglutide $0.75 \mathrm{mg}(54.4 \%)$ and $1.5 \mathrm{mg}$ (55.3\%) compared with insulin glargine (69.1\%), although severe hypoglycemia was rare. At week 52, both doses of dulaglutide were associated with significantly greater improvement compared with insulin glargine in HRQoL (on the EQ-5D), in ability to perform physical activities of daily living (APPADL), and on PROs related to hypoglycemia. ${ }^{29}$
SUSTAIN-4 was a 30-week, multicenter, randomized, openlabel study comparing semaglutide $0.5 \mathrm{mg}$ and $1.0 \mathrm{mg}$ with insulin glargine against a background of metformin with or without sulfonylurea in patients with T2DM. ${ }^{34}$ At 30 weeks, both doses of semaglutide were associated with significantly greater reductions in Alc compared with insulin glargine $(-1.2 \%$ [semaglutide $0.5 \mathrm{mg}$ ], $-1.6 \%$ [semaglutide $1.0 \mathrm{mg}$, $-0.8 \%$ [insulin glargine]). Body weight decreased with both semaglutide doses but increased with insulin glargine $(-3.5 \mathrm{~kg}$ [semaglutide $0.5 \mathrm{mg}$ ], $-5.2 \mathrm{~kg}$ [semaglutide $1.0 \mathrm{mg}$ ]; $+1.2 \mathrm{~kg}$ [insulin glargine]), and the difference was statistically significant $(P<0.0001)$ for both semaglutide doses. Significantly greater percentages of patients developed severe or blood glucose-confirmed hypoglycemia with insulin glargine (11\%) compared with semaglutide $0.5 \mathrm{mg}(4 \%)$ or semaglutide 1.0 $\mathrm{mg}(6 \%)$, although the incidence of severe hypoglycemia was similar and low $(\leq 1 \%)$ in all treatment groups. Both doses of semaglutide were associated with significantly greater overall 


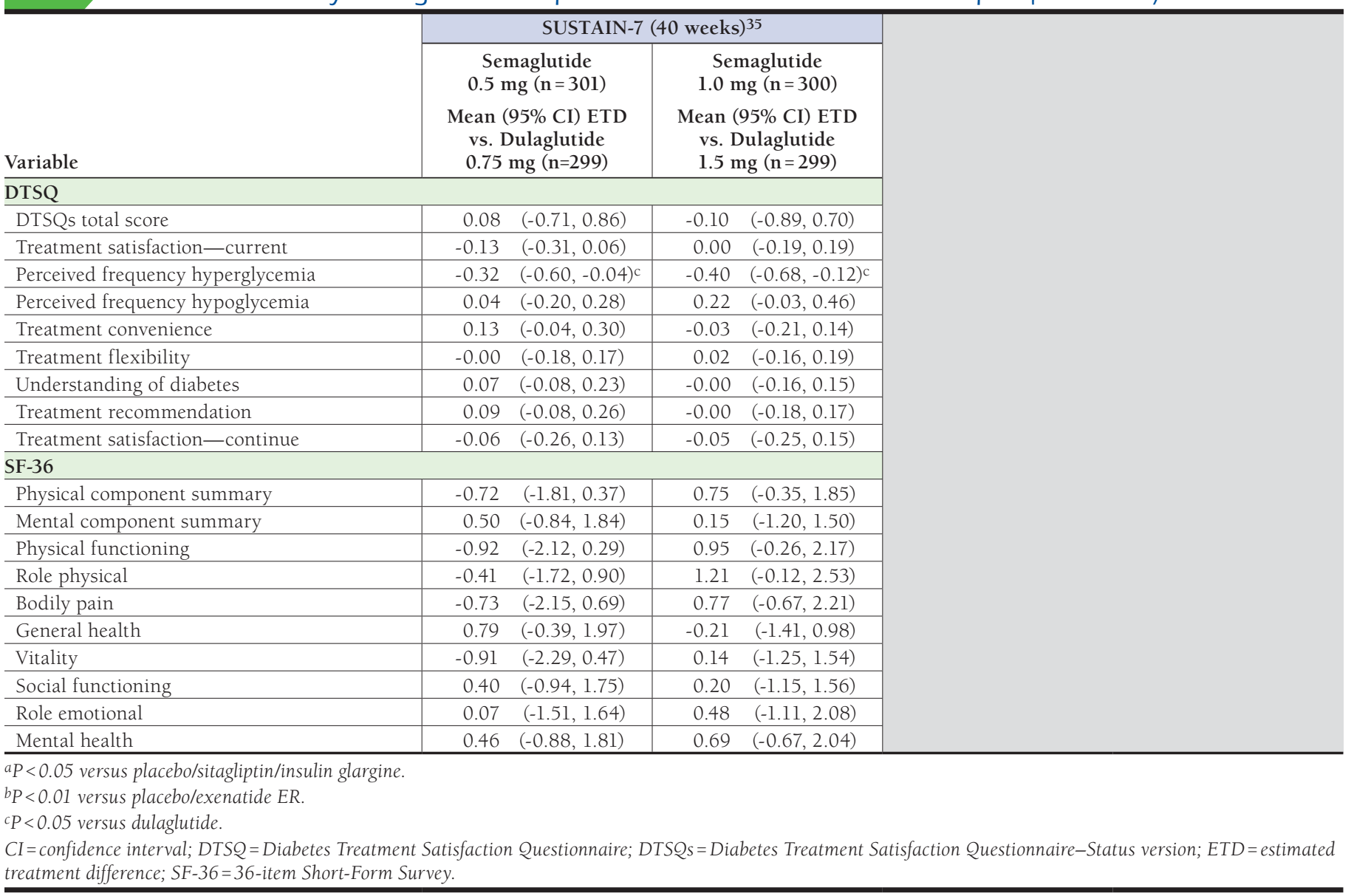

treatment satisfaction than insulin glargine (DTSQ; Table 4). Additionally, the $1.0 \mathrm{mg}$ dose of semaglutide demonstrated significant improvement compared with insulin glargine in the role-emotional (measure of role limitations due to emotional problems) and general health domains of the SF-36 but not on other SF-36 domains.

\section{Once-Weekly Versus Daily GLP-1 RAs}

A 52-week, multicenter, open-label study of patients with T2DM managed with diet and exercise and/or OADs randomized patients to exenatide QW or BID during weeks 1 to 30 (DURATION-1).27,42 Subsequently, all patients received exenatide QW from week 30 to week 52. At 30 weeks, patients receiving exenatide QW had significantly greater reductions in Alc compared with those receiving exenatide BID (-1.9\% vs. $-1.5 \%)$, and a greater proportion of patients treated with exenatide QW achieved Alc $\leq 7 \%$ (77\% vs. $61 \%) .{ }^{42}$ Similar reductions in weight and rates of hypoglycemia were observed with exenatide QW $(-3.7 \mathrm{~kg})$ and exenatide BID $(-3.6 \mathrm{~kg})$. Patients continuing on exenatide QW had sustained reductions in Alc $(2.0 \%$ at week 52), while those who switched from exenatide BID to QW experienced improvements in Alc (-2.0\% at week 52); body weight was similarly reduced in both treatment arms by week 52 (-4.1 $\mathrm{kg}$ [exenatide QW only]; -4.5 kg [exenatide BID to exenatide QW]). ${ }^{43}$ From baseline to week 30, significant improvements in weight-related HRQoL (IWQOL-Lite) and treatment satisfaction (DTSQ) were observed in both treatment groups, with no significant differences between groups (Table 2). ${ }^{27}$ However, at week 30, treatment satisfaction (DTSQ) had improved significantly more in the exenatide QW group than the exenatide BID group with respect to perceived hyperglycemia frequency and willingness to continue current treatment. Patients who switched from exenatide BID to exenatide QW at week 30 experienced improvements from week 30 to week 52 (IWQOL-Lite) in physical function and public distress HRQoL domains as well as in overall treatment satisfaction, treatment convenience, treatment flexibility, and satisfaction with continuing current treatment. Between weeks 30 and 52, those 
patients initially taking exenatide QW reported significant improvements in the public distress domain (IWQOL-Lite) as well as in satisfaction with treatment convenience and flexibility (DTSQ). The development of nausea or injection-site reactions did not moderate the effects of treatment on HRQoL or treatment satisfaction.

In a 32-week, multicenter, randomized, open-label study in patients receiving 1 or more OADs (HARMONY-7), albiglutide $30 \mathrm{mg}$ QW was inferior to liraglutide $1.8 \mathrm{mg}$ once daily (QD) with respect to decrease from baseline in Alc (-0.8\% [albiglutide]; $-1.0 \%$ [liraglutide]) and also produced significantly less weight loss than liraglutide $(-0.6 \mathrm{~kg}$ [albiglutide], $-2.2 \mathrm{~kg}$ [liraglutide]). ${ }^{44}$ Treatment satisfaction scores on the Diabetes Medication Satisfaction Questionnaire improved to a similar degree with both treatments (3.3 for albiglutide, 4.4 for liraglutide, $P=0.207$ ).

AWARD-1 was a 52-week, multicenter, randomized, doubleblind, placebo-controlled study in patients with T2DM that compared dulaglutide $(0.75 \mathrm{mg}$ and $1.5 \mathrm{mg})$ with exenatide BID against a background of metformin and pioglitazone therapy. ${ }^{45}$ At 26 weeks (primary endpoint), both doses of dulaglutide reduced Alc to a significantly greater degree than exenatide BID (-1.3\% [dulaglutide $0.75 \mathrm{mg}$ ], $-1.5 \%$ [dulaglutide $1.5 \mathrm{mg}$ ], $-1.0 \%$ [exenatide]). Compared with exenatide BID $(-1.1 \mathrm{~kg})$, dulaglutide $1.5 \mathrm{mg}(-1.3 \mathrm{~kg})$ produced similar mean weight loss, and dulaglutide $0.75 \mathrm{mg}(+0.2 \mathrm{~kg})$ was associated with significant weight gain. No severe hypoglycemic events occurred with dulaglutide, but 2 were reported with exenatide BID. ${ }^{45}$ Both doses of dulaglutide resulted in significantly greater improvement in treatment satisfaction (DTSQs) compared with exenatide BID at weeks 26 and 52 in AWARD-1, although there were no differences between treatment groups in improvement on HRQoL (EQ-5D), impact of weight on self-perception (IWSP), or impact of weight on ability to perform physical ADL 881 (APPADL; Table 2). ${ }^{29,36}$ At weeks 26 and 52, both doses of dulaglutide were associated with significantly greater reductions in perceived frequency of hyperglycemia (DTSQs-Hyperglycemia) compared with exenatide BID. Perceived frequency of hypoglycemia (DTSQs-Hypoglycemia) increased with exenatide BID but not with dulaglutide, and differences for both doses were significant at weeks 26 and 52. There was no linear association between change in Alc and change in overall treatment satisfaction (DTSQ total score), and there also were no significant correlations between change in overall treatment satisfaction and changes in weight or hypoglycemia incidence. ${ }^{36}$

A 26-week, multicenter, randomized, open-label study (AWARD-6) compared dulaglutide $1.5 \mathrm{mg}$ QW with liraglutide $1.8 \mathrm{mg}$ QD as add-on therapy to metformin in patients with T2DM. ${ }^{46}$ Reduction in Alc with dulaglutide $1.5 \mathrm{mg}$ was noninferior to that of liraglutide $1.8 \mathrm{mg}$ ( $1.4 \%$ for both drugs), although significantly greater weight loss was observed with liraglutide (-3.6 kg vs. $-2.9 \mathrm{~kg}$ with dulaglutide). No severe hypoglycemic events occurred. There were no significant differences between dulaglutide $1.5 \mathrm{mg}$ QW and liraglutide $1.8 \mathrm{mg}$ QD with respect to improvement in HRQoL (EQ-5D), impact of weight on self-perception (IW-SP), or impact of weight on APPADL (Table 3). ${ }^{29}$

\section{Comparison Between Once-Weekly GLP-1 RAs}

A randomized, open-label, multicenter, 56-week trial (SUSTAIN-3) compared semaglutide $1.0 \mathrm{mg}$ with exenatide ER $2.0 \mathrm{mg}$ as add-on therapy to 1 or 2 OADs in patients with T2DM. ${ }^{32}$ Compared with exenatide ER, semaglutide produced significantly greater reductions in Alc and weight at week 56 . Improvement in overall treatment satisfaction was significantly greater with semaglutide versus exenatide ER (Table 4).

SUSTAIN-7 was an open-label, multicenter, 40-week study that compared semaglutide $0.5 \mathrm{mg}$ versus dulaglutide $0.75 \mathrm{mg}$ and semaglutide $1.0 \mathrm{mg}$ versus dulaglutide $1.5 \mathrm{mg}$ as add-on therapy to a stable metformin regimen in adults with T2DM. ${ }^{35}$ Changes from baseline to week 40 in Alc and body weight were significantly greater with semaglutide $0.5 \mathrm{mg}$ versus dulaglutide $0.75 \mathrm{mg}$ (Alc: $-1.5 \%$ [semaglutide $0.5 \mathrm{mg}$ ], $-1.1 \%$ [dulaglutide $0.75 \mathrm{mg}$ ]; body weight: $-4.6 \mathrm{~kg}$ [semaglutide $0.5 \mathrm{mg}$ ], $-2.3 \mathrm{~kg}$ [dulaglutide $0.75 \mathrm{mg}$ ]) and with semaglutide $1.0 \mathrm{mg}$ versus dulaglutide $1.5 \mathrm{mg}$ (Alc: $-1.8 \%$ [semaglutide $1.0 \mathrm{mg}$, $-1.4 \%$ [dulaglutide $1.5 \mathrm{mg}$; body weight: $-6.5 \mathrm{~kg}$ [semaglutide $1.0 \mathrm{mg}$ ], $-3.0 \mathrm{~kg}$ [dulaglutide $1.5 \mathrm{mg}$ ]). Rates of severe or blood glucose-confirmed hypoglycemia were low (1\%-2\%) across treatment groups. Total treatment satisfaction scores (DTSQ) were high at baseline, and changes from baseline in overall treatment satisfaction did not differ between semaglutide and dulaglutide (Table 4). In contrast to the greater benefit of semaglutide on Alc levels, the only DTSQ item on which there was a significant difference involved perceived hyperglycemia, with responses favoring dulaglutide $(P<0.05$; Table 4$)$. HRQoL, as assessed with the SF-36, did not differ between semaglutide and dulaglutide.

\section{Treatment Adherence}

Once-weekly GLP-1 RAs may potentially support greater adherence than daily GLP-1 RAs because of the convenience of less frequent dosing. ${ }^{47}$ Accordingly, studies have demonstrated greater adherence with exenatide $\mathrm{QW}$ versus exenatide BID $^{48,49}$ and liraglutide QD. ${ }^{48-50}$ A retrospective study using U.S. administrative claims data found that, relative to patients receiving exenatide $\mathrm{QW}$, the odds of achieving $\geq 80 \%$ adherence over 6 months were lower with exenatide BID (odds ratio $[O R]=0.41)$ and liraglutide $(O R=0.80$, both $P<0.001)$, and the odds of achieving $\geq 90 \%$ adherence were lower with exenatide BID $(\mathrm{OR}=0.31)$ and liraglutide $(\mathrm{OR}=0.60$, both $P<0.001){ }^{48}$ Similarly, a retrospective cohort study using medical and pharmacy claims data reported that, over 6 months, significantly higher percentages of patients treated with exenatide QW 
had $\geq 80 \%$ adherence (43.2\%) versus exenatide BID (39.0\%, $P<0.01)$ and liraglutide $(35.0 \%, P<0.001)$, and patients receiving exenatide QW were also more likely to have $\geq 90 \%$ adherence $(37.2 \%)$ compared with exenatide BID (20.6\%) or liraglutide (23.3\%; $P<0.001$ for both comparisons). ${ }^{49}$ In an analysis of adherence data from a German prescriptions database, patients receiving exenatide ER were significantly more likely than those receiving liraglutide to achieve $\geq 80 \%$ adherence over 6 months $(\mathrm{OR}=1.78, \mathrm{P}<0.0001) .^{50}$ Greater adherence (percentage achieving $\geq 80 \%$ adherence over 6 months, with propensity score matching used to account for possible selection bias: 54.2\% [dulaglutide] vs. 37.9\% [exenatide QW], $P<0.0001$; $53.5 \%$ [dulaglutide] vs. $44.3 \%$ [liraglutide], $P<0.0001$ ) and persistence (percentage of patients who discontinued treatment, with propensity score matching used to account for possible selection bias: $26.2 \%$ [dulaglutide] vs. $48.4 \%$ [exenatide QW], $P<0.0001 ; 28.0 \%$ [dulaglutide] vs. 35.6\% [liraglutide], $P<0.0001)$ have also been observed with dulaglutide versus exenatide QW or liraglutide QD. ${ }^{51}$ Further research is needed to better define the benefits of once-weekly GLP-1 RAs with regard to HRQoL and treatment satisfaction and to clarify the relationship of these benefits to treatment adherence.

\section{Discussion}

It is important to understand the impact of diabetes treatments on HRQoL given the treatment goal of reducing diabetes-related impairment in HRQoL and considering that treatment-related improvements in HRQoL may enable patients to better manage their self-care and engage in health-promoting activities, such as exercise and healthy diet. Improvements in treatment satisfaction may enhance HRQoL and may also potentially translate to better treatment adherence, which in turn may improve disease control and lead to better health outcomes.

Studies of once-weekly GLP-1 RAs have employed a variety of tools to assess the effects of medications on HRQoL and treatment satisfaction, as well as on various aspects of these PROs. When compared with OADs, once-weekly GLP-1 RAs demonstrated some advantages with regard to glycemic control, weight, and hypoglycemia risk that, in some but not all cases, were associated with significantly greater improvement in corresponding aspects of HRQoL and treatment satisfaction. For example, in DURATION-2, exenatide ER significantly reduced weight compared with both sitagliptin and pioglitazone and also significantly improved weight-related HRQoL versus pioglitazone (but not sitagliptin). ${ }^{28}$ In this study, there was a significant correlation between changes in weight and changes in weight-related HRQoL. AWARD-3 found that dulaglutide improved Alc relative to metformin, and the $1.5 \mathrm{mg}$ dose of dulaglutide was associated with greater improvement in perceived hyperglycemia versus metformin. ${ }^{29,36}$

Insulin has the advantage of theoretically unlimited efficacy but also has several disadvantages, including weight gain and hypoglycemia risk, among others, that may adversely affect HRQoL. ${ }^{7}$ At 52 weeks (AWARD-2), dulaglutide was associated with significantly greater improvement compared with insulin glargine in HRQoL, in ability to perform physical activities of daily living, and on PROs related to hypoglycemia. Similarly, in SUSTAIN-4, semaglutide was associated with greater treatment satisfaction than insulin glargine, and the higher semaglutide dose $(1.0 \mathrm{mg}$ ) also demonstrated significant improvement compared with insulin glargine in the role-emotional and general health domains of the SF-36. ${ }^{34}$ These findings indicate that once-weekly GLP-1 RAs may have some benefits over insulin in relation to HRQoL and treatment satisfaction, although studies are needed to further elucidate the reasons underlying these benefits (i.e., by examining changes in weight-related HRQoL) and to explore whether once-weekly GLP-1 RAs reduce the distress associated with disease management compared with insulin.

It has been hypothesized that the requirement for fewer injections with once-weekly versus daily GLP-1 RAs may increase patient satisfaction and HRQoL.52 Accordingly, in surveys, patients with T2DM have reported a preference for onceweekly over daily dosing. ${ }^{47,53,54}$ Comparison of exenatide ER with exenatide in DURATION-1 identified no significant differences between groups at 30 weeks with respect to changes in weight, weight-related HRQoL, or treatment satisfaction. ${ }^{27}$ However, treatment satisfaction improved more with exenatide ER with regard to perceived hyperglycemia frequency (likely related to differential effects on Alc) and willingness to continue current treatment, which may reflect the convenience of once-weekly treatment. Further, between weeks 30 and 52, patients who switched from exenatide to exenatide ER experienced improvements in physical function and public distress HRQoL domains as well as in overall treatment satisfaction, treatment convenience, treatment flexibility, and satisfaction with continuing current treatment. When additionally considering results of studies comparing dulaglutide with exenatide ${ }^{29,36}$ and dulaglutide or albiglutide with liraglutide,,$^{29,44}$ evidence suggests that there is not a class benefit of improved HRQoL and treatment satisfaction associated with once-weekly GLP-1 RAs; there is, however, a signal that certain elements of treatment satisfaction and willingness to continue treatment may be enhanced with once-weekly GLP-1 RAs.

Only 2 studies (SUSTAIN-3 and SUSTAIN-7) were identified comparing once-weekly GLP-1 RAs with each other with respect to treatment satisfaction ${ }^{32,35}$ and/or HRQoL. ${ }^{35}$ Semaglutide produced significantly greater reductions in Alc and weight than exenatide ER and dulaglutide and was also associated with significantly greater improvement in overall treatment satisfaction compared with exenatide, but not with dulaglutide. ${ }^{32,35}$ There were no significant differences between semaglutide and dulaglutide in HRQoL. ${ }^{35}$ Additional comparative trials are warranted. 


\section{Conclusions and Clinical Implications}

T2DM is associated with considerable impairment in HRQoL. ${ }^{1-4}$ In addition, adverse effects of the medications used to control T2DM, such as weight gain and hypoglycemia, and practical aspects such as cost and administration burden may further impair patients' HRQoL. Thus, as a component of a comprehensive management plan, it is critical to assess and monitor the impact of diabetes and its treatment on HRQoL. Screening for depression (e.g., with the 2-item ${ }^{55}$ or the 9-item Patient Health Questionnaire ${ }^{56}$ ) is increasingly being incorporated into clinical practice, and more systematic assessment of HRQoL and psychological well-being using validated tools, although time-consuming, may enhance overall patient management.

When selecting a medication regimen for patients with T2DM, it is important to consider the impact of treatment on HRQoL. Once-weekly GLP-1 RAs appear to offer benefits with regard to HRQOL and treatment satisfaction compared with OADs, insulin, and daily GLP-1 RAs. The observed benefits are often related to relative drug effects on glycemic control, weight, and hypoglycemia, and their detection is dependent on the use of appropriate assessment tools. Differences in patient populations/background medications and assessment tools employed would make it difficult to interpret any indirect comparisons across studies between once-weekly GLP-1 RAs. Once-weekly GLP-1 RAs represent an effective and convenient treatment option that may potentially enhance treatment adherence, leading to improved health outcomes.

\section{Authors}

LIANA K. BILLINGS, MD, MMSc, NorthShore University Health System, Skokie, Illinois; YEHUDA HANDELSMAN, MD, FACP, FNLA, FASPC, MACE, Metabolic Institute of America, Tarzana, California; MICHAEL HEILE, MD, FAAFP, TriHealth, Cincinnati, Ohio; DORON SCHNEIDER, MD, FACP, Jefferson Health at Abington Hospital, Abington, Pennsylvania; and KATHLEEN WYNE, MD, PhD, FACE, FNLA, The Ohio State University Wexner Medical School, Columbus.

\section{DISCLOSURES}

This supplement was funded by Novo Nordisk. Billings reports personal fees from Dexcom, Novo Nordisk, and Sanofi. Handelsman reports research grants from Amgen, AstraZeneca, Bristol-Myers Squibb, Boehringer Ingelheim, Grifols, Janssen, Lexicon, Merck, Novo Nordisk, Regeneron, and Sanofi; speaker fees from Amarin, Amgen, AstraZeneca, Boehringer Ingelheim-Lilly, Janssen, Merck, Novo Nordisk, Regeneron, and Sanofi; and has served in advisory capacity to Amarin, Amgen, AstraZeneca, Boehringer Ingelheim, Eisai, Intarcia, Janssen, Lilly, Merck, Merck-Pfizer, Novo Nordisk, Regeneron, and Sanofi. Heile reports speaker fees from and has served as advisor to Novo Nordisk. Schneider reports advisory board fees from Intarcia, Lilly, and Novo Nordisk. Wyne has nothing to disclose.

\section{ACKNOWLEDGMENTS}

Writing assistance was provided by Adrienne Drinkwater, $\mathrm{PhD}$, and Sandra Westra, PharmD, through Churchill Communications (Maplewood, NJ) and funded by Novo Nordisk.

\section{REFERENCES}

1. Rubin RR, Peyrot M. Quality of life and diabetes. Diabetes Metab Res Rev. 1999;15(3):205-18

2. Luscombe FA. Health-related quality of life measurement in type 2 diabetes. Value Health. 2000;3(Suppl 1):15-28

3. Centers for Disease Control and Prevention (CDC). Self-rated fair or poor health among adults with diabetes-United States, 1996-2005. MMWR Morb Mortal Wkly Rep. 2006;55(45):1224-127.

4. Grandy S, Fox KM; SHIELD Study Group. Change in health status (EQ5D) over 5 years among individuals with and without type 2 diabetes mellitus in the SHIELD longitudinal study. Health Qual Life Outcomes. 2012;10:99.

5. Grandy S, Chapman RH, Fox KM for the SHIELD Study Group. Quality of life and depression of people living with type 2 diabetes mellitus and those at low and high risk for type 2 diabetes: findings from the Study to Help Improve Early evaluation and management of risk factors Leading to Diabetes (SHIELD). Int J Clin Pract. 2008;62(4):562-68.

6. Trikkalinou A, Papazafiropoulou AK, Melidonis A. Type 2 diabetes and quality of life. World J Diabetes. 2017;8(4):120-29.

7. American Diabetes Association. Standards of medical care in diabetes-2018. Available at: http://diabetesed.net/wp-content/ uploads/2017/12/2018-ADA-Standards-of-Care.pdf. Accessed July 9, 2018.

8. Traina S, Guthrie R, Slee A. The impact of weight loss on weight-related quality of life and health satisfaction: results from a trial comparing canagliflozin with sitagliptin in triple therapy among people with type 2 diabetes. Postgrad Med. 2014;126(3):7-15

9. Wycherley TP, Clifton PM, Noakes M, Brinkworth GD. Weight loss on a structured hypocaloric diet with or without exercise improves emotional distress and quality of life in overweight and obese patients with type 2 diabetes. J Diabetes Investig. 2014;5(1):94-98.

10. Bode BW, Testa MA, Magwire M, et al. Patient-reported outcomes following treatment with the human GLP-1 analogue liraglutide or glimepiride in monotherapy: results from a randomized controlled trial in patients with type 2 diabetes. Diabetes Obes Metab. 2010;12(7):604-12

11. Bergenstal RM, Garrison LP Jr, Miller LA, et al. Exenatide BID Observational Study (ExOS): results for primary and secondary endpoints of a prospective research study to evaluate the clinical effectiveness of exenatide BID use in patients with type 2 diabetes in a real-world setting. Curr Med Res Opin. 2011;27(12):2335-42.

12. Grant P, Lipscomb D, Quin J. Psychological and quality of life changes in patients using GLP-1 analogues. J Diabetes Complications. 2011;25(4):244-46.

13. Reaney M, Mathieu C, Ostenson CG, et al. Patient-reported outcomes among patients using exenatide twice daily or insulin in clinical practice in six European countries: the CHOICE prospective observational study. Health Qual Life Outcomes. 2013;11:217.

14. Ishii H, Niiya T, Ono Y, Inaba N, Jinnouchi H, Watada H. Improvement of quality of life through glycemic control by liraglutide, a GLP-1 analog, in insulin-naive patients with type 2 diabetes mellitus: the PAGEl study. Diabetol Metab Syndr. 2017;9:3.

15. Hayes RP, Schultz EM, Naegeli AN, Curtis BH. Test-retest, responsiveness, and minimal important change of the ability to perform physical activities of daily living questionnaire in individuals with type 2 diabetes and obesity. Diabetes Technol Ther. 2012;14:1118-25. 
16. Meadows KA, Abrams C, Sandbaek A. Adaptation of the Diabetes Health Profile (DHP-1) for use with patients with type 2 diabetes mellitus: psychometric evaluation and cross-cultural comparison. Diabet Med. 2000;17(8):572-80.

17. EuroQol Group. EuroQol-a new facility for the measurement of healthrelated quality of life. Health Policy. 1990;16:199-208.

18. Manwaring J, Wilfley D. The impact of weight on Quality of Life Questionnaire. In: Preedy VR, Watson RR, eds. Handbook of Disease Burdens and Quality of Life Measures. New York: Springer; 2010:210-25

19. Kolotkin RL, Crosby RD, Williams GR. Assessing weight-related quality of life in obese persons with type 2 diabetes. Diabetes Res Clin Pract. 2003;61:125-32.

20. Hayes RP, DeLozier AM. Reliability, validity, and responsiveness of the impact of weight on self-perceptions questionnaire (IW-SP) in individuals with type 2 diabetes and obesity. Diabetes Technol Ther. 2015;17:210-14.

21. Matza LS, Boye KS, Yurgin N. Validation of two generic patient-reported outcome measures in patients with type 2 diabetes. Health Qual Life Outcomes. 2007;5:47.

22. Dupuy HJ. The Psychological General Well-Being (PGWB) Index. In: Wenger NK, ed. Assessment of Quality of Life in Clinical Trials of Cardiovascular Therapies. New York: LeJacq Publications; 1984:170-83.

23. Ware JE. SF-36 health survey update. Spine (Phila Pa 1976). 2000;25(24):3130-39.

24. Rand Health. 36-item Short-Form Survey (SF-36) scoring instructions. Available at: https://www.rand.org/health/surveys_tools/mos/36-item-shortform/scoring.html. Accessed July 9, 2018.

25. Pouwer F, Snoek FJ, van der Ploeg HM, Heine RJ, Brand AN. A comparison of the standard and the computerized versions of the Wellbeing Questionnaire (WBQ) and the Diabetes Treatment Satisfaction Questionnaire (DTSQ). Qual Life Res. 1998;7(1):33-38.

26. Bradley C. The Diabetes Treatment Satisfaction Questionnaire: DTSQ. In: Bradley C, ed. Handbook of Psychology and Diabetes: A Guide to Psychological Measurement in Diabetes Research and Practice. Chur, Switzerland: Harwood Academic Publishers; 1994:111-32.

27. Best JH, Boye KS, Rubin RR, Cao D, Kim TH, Peyrot M. Improved treatment satisfaction and weight-related quality of life with exenatide once weekly or twice daily. Diabet Med. 2009;26(7):722-28.

28. Best JH, Rubin RR, Peyrot M, et al. Weight-related quality of life, health utility, psychological well-being, and satisfaction with exenatide once weekly compared with sitagliptin or pioglitazone after 26 weeks of treatment. Diabetes Care. 2011;34(2):314-19.

29. Yu M, Van Brunt K, Varnado OJ, Boye KS. Patient-reported outcome results in patients with type 2 diabetes treated with once-weekly dulaglutide: data from the AWARD phase III clinical trial programme. Diabetes Obes Metab. 2016;18(4):419-24.

30. Pozzilli P, Norwood P, Jódar E, et al. Placebo-controlled, randomized trial of the addition of once-weekly glucagon-like peptide-1 receptor agonist dulaglutide to titrated daily insulin glargine in patients with type 2 diabetes (AWARD-9). Diabetes Obes Metab. 2017;19(7):1024-31.

31. Rodbard H, Lingvay I, Reed J, et al. Efficacy and safety of semaglutide once-weekly versus placebo as add-on to basal insulin alone or basal insulin in combination with metformin in subjects with type 2 diabetes (SUSTAIN 5). Presented at: European Foundation for the Study of Diabetes (EASD), Berlin, Germany; September 12-16, 2016 [Abstract 766]

32. Ahmann A, Capehorn M, Charpentier G, et al. Efficacy and safety of once-weekly semaglutide vs exenatide ER after 56 weeks in subjects with type 2 diabetes (SUSTAIN 3) [Abstract 187-OR]. Poster presented at: American Diabetes Association 76th Scientific Session; June 10-14, 2016; New Orleans, LA.
33. Ahrén B, Masmiquel L, Kumar H, et al. Efficacy and safety of onceweekly semaglutide versus once-daily sitagliptin as an add-on to metformin, thiazolidinediones, or both, in patients with type 2 diabetes (SUSTAIN 2): a 56-week, double-blind, phase 3a, randomised trial. Lancet Diabetes Endocrinol. 2017;5(5):341-54.

34. Aroda VR, Bain SC, Cariou B, et al. Efficacy and safety of once-weekly semaglutide versus once-daily insulin glargine as add-on to metformin (with or without sulfonylureas) in insulin-naive patients with type 2 diabetes (SUSTAIN 4): a randomised, open-label, parallel-group, multicentre, multinational, phase 3a trial. Lancet Diabetes Endocrinol. 2017;5(5):355-66.

35. Pratley RE, Aroda VR, Lingvay I, et al. Semaglutide versus dulaglutide once weekly in patients with type 2 diabetes (SUSTAIN 7): a randomised, open-label, phase 3b trial. Lancet Diabetes Endocrinol. 2018;6(4):275-86.

36. Reaney M, Yu M, Lakshmanan M, Pechtner V, van Brunt K. Treatment satisfaction in people with type 2 diabetes mellitus treated with once-weekly dulaglutide: data from the AWARD-1 and AWARD-3 clinical trials. Diabetes Obes Metab. 2015;17(9):896-903.

37. Bergenstal RM, Wysham C, Macconell L, et al. Efficacy and safety of exenatide once weekly versus sitagliptin or pioglitazone as an adjunct to metformin for treatment of type 2 diabetes (DURATION-2): a randomised trial. Lancet. 2010;376(9739):431-39.

38. Umpierrez G, Tofé Povedano S, Pérez Manghi F, Shurzinske L, Pechtner V. Efficacy and safety of dulaglutide monotherapy versus metformin in type 2 diabetes in a randomized controlled trial (AWARD-3). Diabetes Care. 2014;37(8):2168-76

39. Nauck M, Weinstock RS, Umpierrez GE, et al. Efficacy and safety of dulaglutide versus sitagliptin after 52 weeks in type 2 diabetes in a randomized controlled trial (AWARD-5). Diabetes Care. 2014;37(8):2149-58.

40. Blonde L, Jendle J, Gross J, et al. Once-weekly dulaglutide versus bedtime insulin glargine, both in combination with prandial insulin lispro, in patients with type 2 diabetes (AWARD-4): a randomised, open-label, phase 3, non-inferiority study. Lancet. 2015;385(9982):2057-66.

41. Giorgino F, Benroubi M, Sun JH, Zimmermann AG, Pechtner V. Efficacy and safety of once-weekly dulaglutide versus insulin glargine in patients with type 2 diabetes on metformin and glimepiride (AWARD-2). Diabetes Care. 2015;38(12):2241-49.

42. Drucker DJ, Buse JB, Taylor K, et al. Exenatide once weekly versus twice daily for the treatment of type 2 diabetes: a randomised, open-label, noninferiority study. Lancet. 2008;372(9645):1240-50.

43. Buse JB, Drucker DJ, Taylor KL, et al. DURATION-1: exenatide once weekly produces sustained glycemic control and weight loss over 52 weeks. Diabetes Care. 2010;33(6):1255-61.

44. Pratley RE, Nauck MA, Barnett AH, et al. Once-weekly albiglutide versus once-daily liraglutide in patients with type 2 diabetes inadequately controlled on oral drugs (HARMONY 7): a randomised, open-label, multicentre, non-inferiority phase 3 study. Lancet Diabetes Endocrinol. 2014;2(4):289-97.

45. Wysham C, Blevins T, Arakaki R, et al. Efficacy and safety of dulaglutide added onto pioglitazone and metformin versus exenatide in type 2 diabetes in a randomized controlled trial (AWARD-1). Diabetes Care. 2014;37(8):2159-67.

46. Dungan KM, Povedano ST, Forst T, et al. Once-weekly dulaglutide versus once-daily liraglutide in metformin-treated patients with type 2 diabetes (AWARD-6): a randomised, open-label, phase 3, non-inferiority trial. Lancet. 2014;384:1349-57.

47. Qin L, Chen S, Flood E, et al. Glucagon-like peptide-1 receptor agonist treatment attributes important to injection-naïve patients with type 2 diabetes mellitus: a multinational preference study. Diabetes Ther. 2017a;8(2):321-34.

48. Johnston SS, Nguyen H, Felber E, et al. Retrospective study of adherence to glucagon-like peptide-1 receptor agonist therapy in patients with type 2 diabetes mellitus in the United States. Adv Ther. 2014;31(11):1119-33. 
49. Nguyen H, Dufour R, Caldwell-Tarr A. Glucagon-like peptide-1 receptor agonist (GLP-1RA) therapy adherence for patients with type 2 diabetes in a Medicare population. Adv Ther. 2017;34(3):658-73.

50. Qiao Q, Ouwens MJ, Grandy S, Johnsson K, Kostev K. Adherence to GLP-1 receptor agonist therapy administered by once-daily or once-weekly injection in patients with type 2 diabetes in Germany. Diabetes Metab Syndr Obes. 2016;9:201-05.

51. Alatorre C, Fernández Landó L, Yu M, et al. Treatment patterns in patients with type 2 diabetes mellitus treated with glucagon-like peptide-1 receptor agonists: higher adherence and persistence with dulaglutide compared with once-weekly exenatide and liraglutide. Diabetes Obes Metab. 2017;19(7):953-61.

52. Zaccardi F, Htike ZZ, Webb DR, Khunti K, Davies MJ. Benefits and harms of once-weekly glucagon-like peptide-l receptor agonist treatments: a systematic review and network meta-analysis. Ann Intern Med. 2016;164(2):102-13.
53. Hauber AB, Nguyen H, Posner J, Kalsekar I, Ruggles J. A discrete-choice experiment to quantify patient preferences for frequency of glucagon-like peptide-1 receptor agonist injections in the treatment of type 2 diabetes. Curr Med Res Opin. 2016;32(2):251-62.

54. Qin L, Chen S, Flood E, et al. Glucagon-like peptide-1 receptor agonist treatment attributes important to injection-experienced patients with type 2 diabetes mellitus: a preference study in Germany and the United Kingdom. Diabetes Ther. 2017;8(2):335-53.

55. Kroenke K, Spitzer RL, Williams JB. The Patient Health Questionnaire-2: validity of a two-item depression screener. Med Care. 2003;41(11):1284-92.

56. Kroenke K, Spitzer RL, Williams JB. The PHQ-9: validity of a brief depression severity measure. J Gen Intern Med. 2001;16(9):606-13. 